\title{
Special issue on DISC 2013, 2014 and PODC 2014
}

\author{
Hagit Attiya ${ }^{1}$
}

Published online: 13 September 2017

(C) Springer-Verlag GmbH Germany 2017

This special issue of Distributed Computing is based on papers that originally appeared as extended abstracts in the Proceedings of the 27th International Symposium on Distributed Computing (DISC 2013), held in Jerusalem, Israel, in the Proceedings of the 28th International Symposium on Distributed Computing (DISC 2014), held in Austin, Texas, and in the Proceedings of the 33rd Symposium on Principles of distributed computing (PODC 2014), held in Paris, France.
The papers for the Special Issue were chosen by the respective Program Committees from the regular papers presented at the symposium, based on their quality and representation of the spectrum of topics encompassed by the Symposium. In addition to being reviewed, in preliminary form, by the Program Committee, the full papers submitted for the Special Issue were refereed according to the standard practices of Distributed Computing. We thank the handling editors, the referees and the authors of these papers.
Hagit Attiya

hagit@cs.technion.ac.il

1 Department of Computer Science, Technion, Haifa, Israel 